Una revisión de los efectos del Tratado de Libre Comercio entre Colombia y Estados Unidos

Gustavo Hernández 
Lecturas de Economía, 80 (enero-junio 2014), pp. 49-77

Gustavo Hernández.

Una revisión de los efectos del Tratado de Libre Comercio entre Colombia y Estados Unidos

Resumen: En la evaluación de politicas arancelarias se han utilizado ampliamente los modelos de equilibrio general computable como herramienta para el análisis y evaluación de escenarios, con el objetivo de conocer los efectos sobre la economía de este tipo de políticas. Este artículo reseña las evaluaciones realizadas al Tratado de Libre Comercio entre Colombia y Estados Unidos (TLC), mostrando las ventajas, desventajas y diferentes alternativas de modelación utilizadas. Se encuentra que la diversidad de los resultados en la evaluación de los posibles efectos del TLC provienen de tres fuentes: la estructura del modelo, las elasticidades. Armington y la agregación sectorial.

Palabras clave: Modelos de Equilibrio General Computable, TLC entre Colombia y Estados Unidos, Política Arancelaria.

Clasificación JEL: C68, D04, F13

Tariff Policy Assessment: An application to the Free Trade Agreement between Colombia and U.S.

Abstract: Computable general equilibrium models have been widely used in tariff policy assessment as a tool for the analysis and evaluation of scenarios, with the aim to ascertain the effects of these policies on the economy. This paper reviews the assessments to the Free Trade Agreement between Colombia and the United States (FTA), showing the advantages, disadvantages and different modeling alternatives. The diversity of the results in the evaluation of the possible effects of the FTA is found to arise from three sources: the structure of the model, the Armington elasticities and sectoral aggregation.

Keywords: Computable general equilibrium models, FTA between Colombia and the U.S., tariff policy

JEL classification: C68, D04, F13

Évaluation de la politique tarifaire: une application pour 1'Accord de Libre-échange entre la Colombie et les États-Unis

Résumé: L'étude des politiques tarifaires ont largement utilisés les modèles d'équilibre général calculable entant qu'un outil d'évaluation des différents scénarios, afin de mieux connaître les effets de ces politiques sur une économie. Cet article passe en revue les évaluations réalisées pour l'Accord de Libre-échange entre la Colombie et les États-Unis (TLC), montrant les avantages, les inconvénients et les alternatives de modélisation qui peuvent être utilisées. Nous constatons que la diversité des évaluations de l'Accord a trois sources: la structure du modèle, les élasticités d'Armington et l'agrégation sectorielle.

Mots-clés: Modèles d'équilibre général calculable, Accord commercial entre la Colombie et les États-Unis, politique tarifaire.

Classification JEL: C68, D04, F13. 
Lecturas de Economía, 80 (enero-junio), pp. 49-77 (C) Universidad de Antioquia, 2014

\title{
Una revisión de los efectos del Tratado de Libre Comercio entre Colombia y Estados Unidos
}

\author{
Gustavo Hernández* \\ -Introducción. -I. Elasticidades de comercio internacional. -II. Impacto del TLC con \\ Estados Unidos. -Comentarios finales. -Bibliografía. -Apéndice. \\ doi: 10.17533/udea.le.n80a2 \\ Primera versión recibida el 15 mayo de 2013; versión final aceptada el 15 de noviembre de 2013
}

\section{Introducción}

Durante los últimos veinte años la política comercial colombiana se ha orientado hacia una mayor apertura comercial y una mayor integración con la economía global. Dentro de los instrumentos de política comercial para lograr estos dos objetivos, se han utilizado los acuerdos de preferencias arancelarias. Dentro de ellos se pueden identificar dos clases: la opción de first best, una disminución unilateral de los aranceles ${ }^{1}$; y la opción de second best ${ }^{2}$, los tratados

* Gustavo Hernández: Subdirector de estudios sectoriales y regulación, Departamento Nacional de Planeación. Dirección postal: calle 26 \# 13-19, piso 18 - Edificio Fonade, Bogotá, Colombia. Dirección electrónica: ghernandez@dnp.gov.co

Los comentarios y errores son responsabilidad del autor y no comprometen a la institución en la que trabaja.

1 Esta primera opción fue utilizada por Colombia a comienzos de los noventa, y se conoció como la "apertura económica".

2 "El teorema establece que si una de las condiciones necesarias para lograr un óptimo de Pareto no es obtenible, las otras, a pesar de ser teóricamente posibles, dejan de ser deseables. En otras palabras, si una de las condiciones para lograr el óptimo de Pareto no es lograble, solo es posible conseguir un óptimo abandonando las otras condiciones. El óptimo así logrado puede ser llamado el Segundo Mejor porque se logra sujeto a un constreñimiento que, por definición, previene el logro de un óptimo de Pareto.” (Lipsey y Lancaster, 1956). 
de libre comercio. Un tratado de libre comercio (TLC) es un compromiso firmado entre diferentes países para eliminar o disminuir los aranceles entre ellos, independientemente de los aranceles fijados con los países que no se encuentran dentro del acuerdo.

Hay diversas metodologías para la evaluación de políticas comerciales: análisis espacial y no espacial; modelos de equilibrio parcial; modelos de equilibrio general de un solo país, dos países o multipaís; y modelos de gravedad ${ }^{3}$, entre los más aplicados. No existe una metodología que sea superior a la otra, todas analizan el mismo problema pero desde diferentes puntos de vista. En este trabajo la discusión se concentra en los Modelos de Equilibrio General Computable (MEGC), como herramienta para la evaluación del TLC entre Estados Unidos y Colombia.

Desde finales de los noventa se comenzó a hablar de una mayor integración comercial con Estados Unidos, nuestro mayor socio comercial y una de las economías más dinámicas en su momento, como motor para un mayor crecimiento de la economía colombiana. Dentro de la preparación de las negociaciones, se realizaron distintos estudios acerca de las consecuencias económicas de este tipo de política, dando una base para las discusiones de las posibilidades y, claro está, de las ventajas y desventajas de la firma de un tratado de este tipo, así como de preparar políticas complementarias que ayudaran a impulsar el tratado. Estos estudios analizaban los resultados desde distintos ángulos (producción, generación de empleo, distribución del ingreso, etc.), con diferentes tipos de estructuras.

Una de las formas de modelación más utilizada ha sido el MEGC, bajo distintas formas de comportamiento de los mercados. Esto ha llevado a encontrar diferentes resultados cuantitativos mas no cualitativos, tales como un mayor crecimiento de la economía, creación de empleo y fortalecimiento del sector industrial, pero, a su vez, una desaceleración del sector agrícola, sin que el orden de los resultados cambie dramáticamente.

Para poder entender esta variedad de resultados cuantitativos, se hizo una lectura de los diferentes estudios para ver las posibles causas de la dis-

3 Véase Cárdenas y García (2004) y Umaña (2011). 
paridad de los resultados. En este orden de ideas, se tratan de responder las siguientes preguntas, las cuales son desarrolladas en las diferentes secciones del artículo: i) ¿Cuál es la respuesta de las importaciones a los precios? Esto es, qué tan importante es la respuesta de las importaciones ante cambios del precio doméstico, ya que el choque se realiza disminuyendo los aranceles; ii) ¿hay influencia de la base de datos en los resultados? Aunque se ha utilizado en la mayoría de los casos la misma base de datos, el manejo de los datos puede cambiar los resultados encontrados; iii) ¿cómo las diferentes alternativas de modelación afectan los resultados? Ya que se han utilizado de diferente forma las estructuras de mercados, como por ejemplo el comportamiento del mercado laboral y la toma de decisiones de las empresas.

Una de las mayores ventajas de los MEGC es la solidez de la microfundamentación. Estos modelos detallan la conducta de los agentes económicos usando los principios de optimización para la elección (mercancías, capital, trabajo, etc.). Además, integran la conducta de todos los agentes de una forma sistemática, que corresponde a la forma en la que los mercados operan desde el punto de vista neoclásico. Sin embargo, hay que considerar que una de las grandes desventajas, dentro del análisis de comercio internacional, es que no se puede observar la creación de nuevos mercados, ya que la estructura comercial del modelo permanece inalterada, mas no los flujos de comercio, ante cambios de la política arancelaria. Otra de las críticas más frecuentes es que los parámetros utilizados son estimados por fuera del modelo, tomados de la literatura o calibrados en un año base. La gran atención prestada a esta técnica para la evaluación de políticas comerciales es que permite llegar a respuestas numéricas al mismo tiempo que se tiene una sólida fundamentación teórica en los resultados. Claro está que los resultados de los MEGC son tan buenos como la especificación del modelo y los datos que sean utilizados.

El trabajo consta de las siguientes secciones: en la primera sección se presentan los aspectos teóricos más relevantes para la interpretación y análisis los resultados de las simulaciones; de esta manera se ve cómo las elasticidades de comercio, particularmente las Armington, afectan de manera importante los efectos sobre bienestar de la economía y, por ende, de variables clave como la producción, exportaciones, importaciones, empleo, etc. En la se- 
gunda sección se presentan de manera esquemática los modelos de equilibrio general más utilizados en el análisis de comercio internacional, para, a continuación, caracterizar los modelos utilizados en Colombia (estructura y bases de datos) para el análisis de un tratado de libre comercio con Estados Unidos, así como los resultados encontrados a partir de las simulaciones. Finalmente se presentan los comentarios finales.

\section{Elasticidades de comercio internacional}

En la construcción de un MEGC es muy importante el proceso de calibración del modelo, porque muestra si la base de datos con que se alimentan las ecuaciones de éste puede reproducir el año base como una solución del modelo. En la práctica, dado el amplio uso de las funciones CES y CobbDouglas, los parámetros claves del modelo, cuyos valores son requeridos antes del procedimiento de calibración, son más o menos sinónimos de elasticidades. Los resultados de las simulaciones dependen en gran parte de las elasticidades utilizadas, en el caso particular de políticas comerciales son las elasticidades de comercio las más relevantes, ya que determinan la sensibilidad del modelo.

En la modelación del sector de comercio, particularmente de las importaciones, se asume el supuesto Armington. En este contexto, los modelos asumen que las ofertas de un bien particular de origen doméstico son idénticas entre sí, como también todas las fuentes de comercio exterior de este bien, desde el punto de vista del comprador. Entonces, la absorción doméstica consiste en la demanda total de un producto doméstico e importado agregado de un bien en particular, donde la combinación de estas dos mercancías se determina por el precio relativo y el grado de sustitución.

\section{A. ¿Por qué son tan importantes las elasticidades de importaciones?}

Los efectos sobre bienestar de la política arancelaria van a estar determinados por la elasticidad de sustitución entre los bienes domésticos e importados. Para simplificar el problema se realiza el análisis bajo un modelo de equilibrio parcial, manteniéndose los resultados en un análisis de equilibrio 
general. Vamos a considerar el caso de una liberalización unilateral. En el Gráfico 1 puede verse en el panel izquierdo la oferta y demanda del mercado doméstico, y en el panel derecho la oferta y demanda de importaciones. $\mathrm{Al}$ comienzo hay un precio internacional $P^{*}$ al cual se le impone un arancel advalorem, $\tau$. Por lo tanto, el precio doméstico es $P=P^{*}(1+\tau)$, el cual es tomado por la demanda y la oferta doméstica. La diferencia entre la oferta doméstica, $Q^{s}$, y la demanda doméstica, $Q^{d}$, son las importaciones, $M$. Estas importaciones $M$ se pueden observar en el panel derecho, donde está la oferta de importaciones (esto es, la demanda de exportaciones del resto del mundo), $M^{s}$, y la demanda de importaciones, $M^{d}$, y el equilibrio se alcanza en el punto A.

Gráfico 1. Efecto de una reforma unilateral en equilibrio parcial

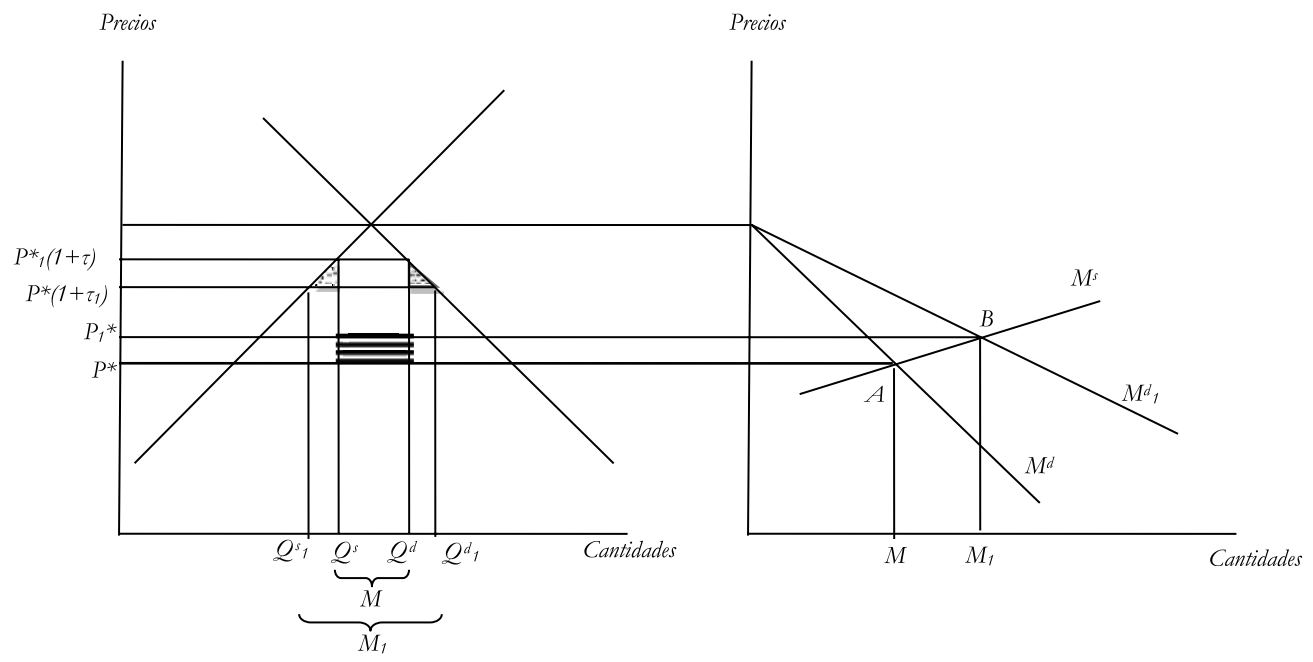

Fuente: adaptación de Bouët (2008).

La eliminación de los aranceles implica que la demanda de importaciones se mueva hacia la derecha, donde las nuevas importaciones son $M 1$. Ahora bien, dado que hay un desbalance entre la oferta y la demanda de importaciones, el precio mundial se incrementa un poco, hasta $\mathrm{P}_{1}{ }^{*}$, de acuerdo a la elasticidad de la oferta de importaciones, para que se encuentre el mercado en equilibrio, esto es en el punto B. El efecto neto es la diferencia entre las áreas punteadas (ganancia en eficiencia) y el área de líneas horizontales (pérdida 
en los términos de intercambio ${ }^{4}$ ). Dado que las exportaciones del resto del mundo tienen una elasticidad muy alta, los precios mundiales se incrementan poco. Esto es bueno para un país importador, ya que el efecto negativo de la liberalización es pequeño; sin embargo, las importaciones son pagadas por el país a un precio más alto, esta pérdida es $\left(P_{1}^{*}-P^{*}\right)\left(Q_{d}-Q_{s}\right)$.

En el caso de que la elasticidad sea más baja, lo cual implicaría que la pendiente de $M^{s}$ sea más vertical, la liberalización comercial produce un pequeño incremento en las importaciones pero un gran aumento de los precios, lo cual implica menores ganancias en bienestar. El efecto sobre bienestar se obtiene a través de dos mecanismos: i) un incremento en las importaciones, lo cual refleja un desplazamiento de la producción nacional por la producción extranjera y un mayor consumo doméstico por unos precios domésticos más bajos, lo que conlleva a un incremento en el recaudo arancelario (efecto positivo) por mayores importaciones, pero a unas menores tasas arancelarias (efecto negativo), sobre lo cual no se puede determinar un efecto a priori ni determinar cuál es su signo; ii) un cambio en los precios internacionales, ya que cuando un producto es importado y se reducen los aranceles se incrementan los precios internacionales, situación que produce un efecto negativo sobre el bienestar.

En el caso de una liberalización multilateral, los efectos sobre los términos de intercambio por un incremento de las exportaciones, ocasionado por un mayor acceso a los mercados internacionales, cambia. Entonces, los exportadores van a tener un mayor precio por sus productos debido a la liberalización de los otros países.

\section{B. Elasticidades de importaciones (Armington) para Colombia}

En una economía abierta, cada mercancía puede ser diferenciada de acuerdo con su fuente de producción: doméstica o importada. Para modelar este hecho, en el comercio internacional desde el artículo seminal de Armington (1969), se supone que entre categorías de bienes existen posibilidades de sustitución entre los bienes importados y los bienes domésticos; por lo tanto,

4 En este caso porque los precios de las importaciones suben. 
el grado de sustitución entre los bienes domésticos y los importados se captura por la elasticidad Armington. Un alto valor de este parámetro significa que el bien importado y el bien ofrecido domésticamente se consideran como virtualmente idénticos por los compradores. Por el contrario, un valor bajo de la elasticidad de sustitución significa que los productos son diferenciados para los compradores, es decir, ellos exhiben una sustitución débil.

Existen tres fuentes de elasticidades de comercio que son las más utilizadas en los diversos modelos multipaís. La primera fuente es Hertel, et al. (2004), quienes estimaron unas elasticidades muy altas para estos parámetros, en promedio es de 7 , lo cual es bastante grande si se compara con las antiguas estimaciones utilizadas por el modelo GTAP, que se encuentra alrededor de 5,3. De otra parte, en los trabajos de Anderson, van der Mensbrugghe y Martin $(2005$; 2006), se muestra que para el modelo LINKAGE las elasticidades utilizadas son una tercera parte que las elasticidades encontradas por Hertel et al. (2004). Finalmente, Harrison, Rutherford y Tarr (1997; 2003), con el modelo GTAPinGAMS utilizan unas elasticidades de 2, las cuales son bastante bajas comparadas con los demás autores.

McDaniel y Balistreri (2003), después de una revisión exhaustiva de la literatura encuentran que: i) las elasticidades de largo plazo son más altas que las estimaciones de corto plazo; ii) los análisis más desagregados encuentran elasticidades mayores; iii) los análisis basados en formas reducidas y series temporales detectan elasticidades menores en relación con los estudios de corte transversal que consideran también las condiciones de oferta.

Para Colombia se han realizado diversas estimaciones de estas elasticidades, bajo diferentes específicas econométricas 5 . Lozano (2004), mediante técnicas de cointegración estima las elasticidades de sustitución Armington, tanto para corto como para largo plazo, encontrando que en el corto plazo varían entre 0,32 y 0,85, similares a las magnitudes reportadas por Hernández (1998), que se encuentran entre las magnitudes de 0,1 y 0,9, sin contar con el sector minero. En cuanto a las elasticidades de largo plazo, Lozano (2004) reporta valores entre 0,3 y 2,1 , con un promedio de 1,05 , un poco más bajas

5 Véase Apéndice para los resultados de las estimaciones. 
Gustavo Hernández: Una revisión de los efectos del Tratado de Libre Comercio...

que las estimadas por Devarajan, Go y Li (1999), que están en promedio en 1,21. En cuanto a las elasticidades para el sector agrícola, Ramírez, et al. (2004) encuentran que las elasticidades han fluctuado entre 2,17 y 0,37 , con un promedio de 1,41 para el sector agropecuario, casi el doble más alto que lo encontrado para este sector por Hernández (1998) y por Lozano (2004), que en promedio son del orden de 0,62 y 0,58 , respectivamente.

\section{Impacto del TLC con Estados Unidos}

Antes de comenzar con el análisis de los resultados encontrados, se deben poner en contexto las simulaciones realizadas. Los diferentes estudios que evaluaron los efectos de un TLC con Estados Unidos también realizaron ejercicios de comparación de lo que sucedía ante la firma del $\mathrm{ALCA}^{6}$ y la renovación del ATPA, conocido posteriormente como ATPDEA7?.

El ALCA y el ATPDEA son dos tipos de políticas arancelarias adoptadas por parte de Estados Unidos, por lo cual Colombia no podía desligarse de estos dos aspectos dentro de la negociación de un TLC con Estados Unidos. La primera no se logró concretar, pero quería conformar un solo bloque comercial para el continente americano, con excepción de Cuba. Por esta razón

6 El ALCA fue el nombre con que se conoció la iniciativa para realizar un TLC entre América del Norte (Canadá, Estados Unidos y México, que ya habían firmado el NAFTA) y el resto de los países del continente americano - excluyendo a Cuba-, que comenzó la etapa de conversaciones en 1994, pero después de muchas rondas, y la falta de compromiso político, fue marchitándose hasta desaparecer en el 2005.

7 La Ley de Preferencias Arancelarias Andinas (ATPA, por sus siglas en inglés) es un régimen de excepción otorgado unilateralmente por los Estados Unidos a Perú, Bolivia, Colombia y Ecuador para apoyar la lucha contra el tráfico ilícito de drogas. Esta comenzó el 4 de diciembre de 1991 ofreciendo ingreso libre de aranceles a cerca de 5.500 productos, donde no se encontraban incluidos, entre otros, los textiles y las confecciones, y venció el 4 de diciembre del 2001. Luego de tres años de trabajo, el Congreso de los Estados Unidos aprobó un nuevo texto renovando y ampliando los beneficios del ATPA, que ahora pasará a ser llamada Ley de Promoción Comercial Andina y Erradicación de la Droga (ATPDEA, por sus siglas en inglés) y terminó a finales de 2011, dando paso a la aprobación del TLC por parte del congreso de los Estados Unidos, negociado con Colombia en el primer semestre del 2012. 
varios de los estudios tratan de mostrar cuál podría ser la mejor estrategia a seguir, si entrar a negociar primero un TLC con Estados Unidos o una negociación del ALCA. En el caso de la segunda política, el ATPDEA era una política de cero aranceles para algunos productos de los países andinos comprometidos con la lucha antidroga, que perdería vigencia para finales del 2001, por lo que cualquier tipo de negociación arancelaria con Estados Unidos estaba ligada a la prorrogación de esta política hasta la entrada en vigencia del TLC con Estados unidos, para que los productores nacionales no asumieran mayores pérdidas.

Como se puede apreciar en el Tabla 1, los estudios han realizado las simulaciones de firmar un TLC con Estados Unidos o el ALCA, tomando como referencia una desgravación arancelaria de forma unilateral por parte de Colombia. Otras políticas que han sido evaluadas, pero con menor atención, han sido: los efectos de la inversión extrajera directa a partir de una liberalización del sector de los servicios, y la eliminación o reducción de barreras no arancelarias.

Tabla 1. Efectos de politica comerciales en equilibrio general computable

\begin{tabular}{|c|c|c|}
\hline Autores & Simulaciones & Principales resultados \\
\hline $\begin{array}{c}\text { Bussolo y } \\
\text { Roland-Holst } \\
\text { (1999) }\end{array}$ & $\begin{array}{l}\text { Eliminación de aranceles bilaterales y barreras } \\
\text { no arancelarias para Estados Unidos, Canadá y } \\
\text { México. } \\
\text { Eliminación de aranceles bilaterales y barreras no } \\
\text { arancelarias para los cuatro países }\end{array}$ & $\begin{array}{l}\text { Colombia se beneficia del TLCAN } \\
\text { aunque no ingrese directamente. } \\
\text { El ingreso de Colombia al TLCAN } \\
\text { es más expansivo que contractivo. }\end{array}$ \\
\hline $\begin{array}{c}\text { Dirección } \\
\text { de Estudios } \\
\text { Económicos } \\
\text { y Dirección } \\
\text { de Desarrollo } \\
\text { Empresarial } \\
(2003)\end{array}$ & $\begin{array}{l}\text { Acuerdo bilateral de libre comercio entre Colom- } \\
\text { bia y Estados Unidos. } \\
\text { Estados Unidos otorga un trato preferencial a las ex- } \\
\text { portaciones provenientes de todos los sectores produc- } \\
\text { tivos de los países beneficiados con el ATPDEA } \\
\text { Colombia no firma un acuerdo bilateral con Es- } \\
\text { tados Unidos, no goza de las preferencias ATP- } \\
\text { DEA y no firma el ALCA. } \\
\text { La zona económica de Centro América pacta un } \\
\text { acuerdo bilateral de libre comercio con Estados } \\
\text { Unidos mientras que Colombia no tiene las pre- } \\
\text { ferencias ATPDEA, no firma el ALCA y tampoco } \\
\text { un bilateral con Estados Unidos. } \\
\text { Todos los países del hemisferio pactan el ALCA. }\end{array}$ & $\begin{array}{l}\text { Las preferencias arancelarias tipo } \\
\text { ATPDEA ofrecen ventajas para los } \\
\text { productos colombianos pero el efec- } \\
\text { to de un acuerdo bilateral con Esta- } \\
\text { dos Unidos es superior porque mejo- } \\
\text { ra la producción y la competitividad } \\
\text { de los sectores en los cuales tenemos } \\
\text { ventajas comparativas. } \\
\text { No participar en acuerdo comercial } \\
\text { alguno deteriora la competitividad } \\
\text { de la economía colombiana y nues- } \\
\text { tros competidores obtienen las ga- } \\
\text { nancias derivadas de la suscripción } \\
\text { de acuerdos comerciales. }\end{array}$ \\
\hline
\end{tabular}

(Continúa) 
Gustavo Hernández: Una revisión de los efectos del Tratado de Libre Comercio...

\section{Tabla 1. (Continuación)}

\begin{tabular}{|c|c|c|}
\hline Autores & Simulaciones & Principales resultados \\
\hline $\begin{array}{l}\text { Light y Ruther- } \\
\text { ford (2003) }\end{array}$ & $\begin{array}{l}\text { Total acceso entre los mercados de América del } \\
\text { norte y América del Sur (ALCA). } \\
\text { ALCA sin Estados Unidos. } \\
\text { ALCA sin concesiones agrícolas. } \\
\text { ATPDEA. } \\
\text { Reducción unilateral de } 50,0 \% \text { de los aranceles } \\
\text { de Colombia. } \\
\text { Área de libre comercio en Sur América }\end{array}$ & $\begin{array}{l}\text { El ALCA produciría un pequeño } \\
\text { incremento en el bienestar de Co- } \\
\text { lombia, que se contrarrestaría si no } \\
\text { hay concesiones agrícolas. } \\
\text { De otra parte el no entrar al ALCA } \\
\text { por parte de Colombia produciría } \\
\text { una disminución del bienestar. }\end{array}$ \\
\hline $\begin{array}{c}\text { Hernández y } \\
\text { Sanchez (2004) }\end{array}$ & $\begin{array}{l}\text { Reducción de } 50 \% \text { en los aranceles. } \\
\text { Incremento en los aranceles de } 50 \% \\
\text { Aumento de } 10 \% \text { en los subsidios a las expor- } \\
\text { taciones. } \\
\text { Remoción total de los aranceles de la agricultura, } \\
\text { la industria y el sector servicios para los países } \\
\text { pertenecientes al ALCA. } \\
\text { Abolición de los aranceles para todos los países } \\
\text { de la } \\
\text { OMC. } \\
\text { Devaluación del tipo de cambio de } 10 \% \text {. }\end{array}$ & $\begin{array}{l}\text { Las políticas de reducción de aran- } \\
\text { celes e incentivos a las exportaciones } \\
\text { favorecen el crecimiento económico } \\
\text { y no tienen impactos negativos so- } \\
\text { bre la distribución. } \\
\text { Los resultados muestran que las po- } \\
\text { líticas de liberalización e integración } \\
\text { comercial reducen la pobreza a nivel } \\
\text { urbano, rural y nacional y que este es } \\
\text { mucho más pronunciado cuando se } \\
\text { analiza el impacto conjunto tanto de } \\
\text { los mayores niveles salariales como } \\
\text { del mayor nivel de empleo. }\end{array}$ \\
\hline Light (2004) & $\begin{array}{l}\text { Eliminación de aranceles y barreras no arancela- } \\
\text { rias para la IED. } \\
\text { Eliminación de } 50,0 \% \text { en los aranceles y barreras } \\
\text { no arancelarias para la IED. } \\
\text { Eliminación de barreras no arancelarias para la } \\
\text { IED pero se mantienen los aranceles. }\end{array}$ & $\begin{array}{l}\text { Incremento del PIB en } 3.2 \% \text { en el corto } \\
\text { plazo y de hasta } 5.8 \% \text { en el largo plazo. } \\
\text { Efecto mucho mayor de la elimina- } \\
\text { ción de las barreras no arancelarias } \\
\text { sobre la IED que de los aranceles } \\
\text { sobre bienes. } \\
\text { Un incremento mínimo del consu- } \\
\text { mo debido a una reducción de los } \\
\text { precios pero mayor competencia } \\
\text { con las empresas americanas. }\end{array}$ \\
\hline Botero (2005) & $\begin{array}{l}\text { Reducción de los aranceles entre Colombia y Es- } \\
\text { tados Unidos. } \\
\text { Total acceso entre los mercados de América del } \\
\text { norte y América del Sur (ALCA). }\end{array}$ & $\begin{array}{l}\text { El TLC generaría un incremento } \\
\text { del PIB del orden del 4\%; en tan- } \\
\text { to que el ALCA representaría un } \\
\text { incremento del } 5,7 \% \text {. Con ello, el } \\
\text { empleo se incrementaría, respecti- } \\
\text { vamente, en } 1,7 \% \text { y } 2,4 \% \text {. }\end{array}$ \\
\hline $\begin{array}{l}\text { López y Botero } \\
\text { (2005) }\end{array}$ & $\begin{array}{l}\text { Eliminación reciproca de los aranceles (Estados } \\
\text { Unidos y Colombia) }\end{array}$ & $\begin{array}{l}\text { Incremento de la mano de obra ca- } \\
\text { lificada. } \\
\text { Impulso al crecimiento de la econo- } \\
\text { mía colombiana. }\end{array}$ \\
\hline
\end{tabular}

(Continúa) 
Tabla 1. (Continuación)

\begin{tabular}{|c|c|c|}
\hline Autores & Simulaciones & Principales resultados \\
\hline $\begin{array}{l}\text { Pardo, et al. } \\
\quad(2005)\end{array}$ & $\begin{array}{l}\text { El TLC consiste en eliminar las tarifas arancela- } \\
\text { rias del comercio de Colombia, Perú y Ecuador } \\
\text { con respecto a los Estados Unidos. }\end{array}$ & $\begin{array}{l}\text { Leve mejoría en los indicadores de } \\
\text { pobreza, sin que exista un deterioro } \\
\text { en la distribución del ingreso. } \\
\text { Ausencia de grandes ganancias para } \\
\text { el país en términos agregados y de } \\
\text { distribución del ingreso. }\end{array}$ \\
\hline $\begin{array}{l}\text { Ramírez y Mar- } \\
\text { tín (2005) }\end{array}$ & $\begin{array}{l}\text { Disminución de barreras arancelarias y no aran- } \\
\text { celarias, manteniendo en algunos casos las del } \\
\text { sector agrícola. }\end{array}$ & $\begin{array}{l}\text { Los efectos del TLC con Estados Uni- } \\
\text { dos dependen críticamente de la dismi- } \\
\text { nución de las barreras no arancelarias. } \\
\text { Efectos distributivos negativos si las } \\
\text { barreras no arancelarias se mantienen. }\end{array}$ \\
\hline Vaughan (2005) & $\begin{array}{l}\text { Eliminación de la barreras arancelarias y no aran- } \\
\text { celarias con Estados Unidos }\end{array}$ & $\begin{array}{l}\text { Ganancias en los sectores en donde } \\
\text { hay una gran protección arancelaria. } \\
\text { Si Estados Unidos eliminara todas } \\
\text { sus barreras no arancelarias ante el } \\
\text { mundo, entonces Colombia podría } \\
\text { tener efectos negativos de comercio. }\end{array}$ \\
\hline Light (2006) & $\begin{array}{l}\text { Eliminación de aranceles y barreras no arancela- } \\
\text { rias para la IED. } \\
\text { Eliminación de } 50,0 \% \text { en los aranceles y barreras } \\
\text { no arancelarias para la IED. } \\
\text { Eliminación de barreras no arancelarias para la } \\
\text { IED pero se mantienen los aranceles. }\end{array}$ & $\begin{array}{l}\text { Particularmente para Colombia: } \\
\text { en mayor parte del incremento de } \\
\text { la inversión extranjera directa, y en } \\
\text { menor grado de la eliminación de } \\
\text { aranceles. } \\
\text { La eliminación de los aranceles andi- } \\
\text { nos tiene una mezcla de efectos: los } \\
\text { precios para bienes finales e insumos } \\
\text { intermedios caen, ayudando a incre- } \\
\text { mentar el estándar de vida, pero los } \\
\text { productores enfrentarán un incremen- } \\
\text { to de las competencias de Estados Uni- } \\
\text { dos, los cuales bajarían la producción e } \\
\text { incrementaría el desempleo. }\end{array}$ \\
\hline $\begin{array}{l}\text { Fedesarrollo } \\
\quad(2007)\end{array}$ & $\begin{array}{l}\text { Liberalización total, aranceles colombianos como } \\
\text { estadounidenses iguales a cero } \\
\text { Efecto del TLC con Estados Unidos sobre la re- } \\
\text { gión en un horizonte de tiempo de } 10 \text { años } \\
\text { No se firma el TLC y se pierden las preferencias } \\
\text { arancelarias actuales }\end{array}$ & $\begin{array}{l}\text { Las mayores fuentes de crecimiento se } \\
\text { encuentran asociadas a aquellos sec- } \\
\text { tores con una mayor orientación ex- } \\
\text { portadora hacia Estados Unidos (por } \\
\text { ejemplo la industria manufacturera) y } \\
\text { hacia el resto del país (por ejemplo, el } \\
\text { sector que contabiliza la actividad de } \\
\text { los establecimientos financieros). }\end{array}$ \\
\hline
\end{tabular}

(Continúa) 
Gustavo Hernández: Una revisión de los efectos del Tratado de Libre Comercio...

Tabla 1. (Continuación)

\begin{tabular}{|c|c|c|}
\hline Autores & Simulaciones & Principales resultados \\
\hline $\begin{array}{c}\text { Gracia y Zuleta } \\
\text { (2007) }\end{array}$ & $\begin{array}{l}\text { Estados Unidos fija un arancel de cero para to- } \\
\text { dos los bienes originarios de los países andinos } \\
\text { (ATPDEA). } \\
\text { Acuerdo bilateral de libre comercio entre Co- } \\
\text { lombia y Estados Unidos, manteniendo las pre- } \\
\text { ferencias del ATPDEA para el resto de los países } \\
\text { andinos. } \\
\text { Acuerdo bilateral de libre comercio entre Estados } \\
\text { Unidos y Colombia, eliminando las preferencias } \\
\text { del ATPDEA para el resto de los países andinos. } \\
\text { Eliminación de todas las barreras comerciales al } \\
\text { interior de América, tomando el ATPDEA como } \\
\text { punto de partida (ALCA). }\end{array}$ & $\begin{array}{l}\text { Aumentos moderados en el bienes- } \\
\text { tar y en la producción de los consu- } \\
\text { midores y firmas colombianos. } \\
\text { Efectos pequeños explicados por- } \\
\text { que ya se tienen preferencias aran- } \\
\text { celarias, particularmente, con Vene- } \\
\text { zuela y Estados Unidos. }\end{array}$ \\
\hline
\end{tabular}

Fuente: elaboración del autor.

\section{A. Estructura de los modelos}

Conocer la estructura del modelo es importante, ya que muestra cómo se pueden involucrar las simulaciones de política y los canales de transmisión de éstas (Tabla 2). La forma de modelización puede ser caracterizada en dos tipos, con algunas variaciones: i) modelos de un solo país y ii) modelos multipaís. En la primera categoría se utiliza un modelo estándar de equilibrio general ${ }^{8}$, donde algunos recurren a desagregar los hogares para ver los efectos sobre pobreza y distribución del ingreso, o incluyen rendimientos crecientes a escala, con el objetivo de analizar las barreras no arancelarias, particularmente en el sector de servicios. En cuanto al modelo multipaís que se utiliza en general se sigue la estructura del modelo GTAPinGAMS ${ }^{9}$ o del modelo GTAP ${ }^{10}$, aunque algunos autores han desarrollado su propio modelo multipaís (Bussolo y Roland-Holst, 1999).

8 Véase Perdomo (2008) o Lofgren, Lee y Robinson (2002) para una detallada descripción de un modelo de equilibrio general computable estándar y sus códigos de programación.

9 Para mayor información consultar Rutherford y Paltsev (2000).

10 Para una descripción detallada del modelo Hertel (1999). 
Tabla 2. Caracteristicas de los modelos

\begin{tabular}{llllc}
\hline \multicolumn{1}{c}{ Autores } & $\begin{array}{c}\text { Tipo de } \\
\text { Modelo }\end{array}$ & $\begin{array}{c}\text { Conducta } \\
\text { de las empresas }\end{array}$ & Dinámica & $\begin{array}{c}\text { Micro } \\
\text {-simulaciones }\end{array}$ \\
\hline Hernández y Sánchez (2004) & Un solo país & Competencia perfecta & No & Si \\
López y Botero (2005) & Un solo país & Competencia perfecta & No & No \\
Fedesarrollo (2007) & Un solo país & Competencia perfecta & Si & No \\
Light ( 2004) & Un solo país & Competencia imperfecta & No & No \\
Botero (2005) & Un solo país & Competencia imperfecta & No & No \\
Ramírez y Martín (2005) & Un solo país & Competencia imperfecta & No & No \\
Bussolo y Roland-Holst (1999) & Multipaís & Competencia perfecta & No & No \\
DEE y DDE (2003) & Multipaís & Competencia perfecta & No & No \\
Light y Rutherford (2003) & Multipaís & Competencia perfecta & No & No \\
Pardo, et al. (2005) & Multipaís & Competencia perfecta & No & Si \\
Vaughan (2005) & Multipaís & Competencia perfecta & No & No \\
Gracia y Zuleta (2007) & Multipaís & Competencia perfecta & No & No \\
Light ( 2006) & Multipaís & Competencia imperfecta & No & No \\
\hline
\end{tabular}

Fuente: elaboración del autor.

Adicionalmente, hay dos formas de modelar la conducta de las empresas: competencia perfecta y competencia imperfecta. La primera, la más utilizada, tiene como supuesto los rendimientos constantes a escala; sin embargo, ignora potenciales ganancias sobre el comercio, como las economías de escala. Para superar este obstáculo se puede asumir la competencia imperfecta, de esta manera se pueden observar las ganancias provenientes de mayor variedad de productos e incrementos en la productividad. Claro está que la mayor desventaja de utilizar este tipo de supuestos proviene de la mayor demanda de datos, los cuales no se encuentran disponibles para toda la economía, por esta razón los modelos se han centrado en aplicar este tipo de estructura sobre el sector industrial.

Para analizar los efectos de las políticas comerciales sobre pobreza y distribución del ingreso, se ha utilizado la técnica de interrelacionar un MEGC con un modelo de microsimulaciones. Los modelos de microsimulaciones permiten observar el proceso mediante el cual el comportamiento de los ho- 
gares o individuos se ve afectado por cambios de política económica. En este caso, se centra en el análisis de las remuneraciones laborales y de la elección ocupacional de los miembros del hogar, para de esta manera poder hacer inferencia sobre los efectos de las políticas comerciales en el mercado laboral, la distribución del ingreso y la pobreza ${ }^{11}$. Hay que aclarar que las microsimulaciones no son efectuadas dentro del modelo, sino que las simulaciones del MEGC son involucradas dentro de un modelo econométrico, tomando como referencia los efectos sobre salarios de la política arancelaria.

\section{B. Datos de los modelos}

Los modelos han utilizado como bases de datos para sus simulaciones la matriz de contabilidad social (MCS), que se ha ido actualizando de acuerdo a la aparición de nuevos datos en las cuentas nacionales, en el caso de los modelos de un solo país, y de la base de datos GTAP ${ }^{12}$, en el caso de modelos multipaís. La base de datos GTAP ha sido un esfuerzo patrocinado por el Departamento de Agricultura de la Universidad de Purdue, que tiene como objetivo mejorar la calidad de la información para la evaluación cuantitativa del comercio internacional bajo un contexto económico ${ }^{13}$. Esta base de datos contiene la descripción de los datos globales acerca de trayectorias bilaterales de comercio, producción, consumo y consumo intermedio de bienes y servicios, para diferentes países o regiones. Esta base cuenta, en su última versión (v. 7.0), con 113 regiones, 57 sectores y como año base el 2004.

Para la utilización de los modelos GTAPinGAMS o GTAP hay que tener en cuenta dos aspectos importantes relacionados con la base de datos: i) para efectos prácticos, tanto computacionales como de interpretación, los modelos deben manejar un bajo grado de agregación, tanto sectorial como regional; y ii) la base de datos, aunque es consistente, tiene un rezago importante

11 Véase Bourguignon, Robilliard y Robinson (2005) para una detallada explicación de la metodología.

12 Global Trade Analysis Project.

13 Para mayor información puede visitar: https://www.gtap.agecon.purdue.edu/. 
con respecto al año en el que se hace la simulación, alrededor de seis a ocho años, por lo que esta debe actualizarse.

Como se mencionó, en primer lugar, debe existir algún grado de agregación porque, desde el punto de vista computacional, al realizar una simulación con todas las regiones y sectores tomaría una gran cantidad de tiempo, además de no poder garantizar la convergencia del modelo. Ahora bien, desde el punto de vista práctico, una región, en este caso Colombia, no tiene necesariamente comercio con todos los países del mundo, o con algunas regiones este puede llegar a ser mínimo, por tanto, al adaptar la base de datos se debe tener en cuenta tanto los principales socios comerciales, como los países o regiones que se van a considerar en el momento de la simulación, para luego realizar la agregación pertinente. Cada uno de los estudios analizados pudo haber utilizado la misma base de datos, pero considerando una agregación diferente, sectorial y regionalmente, de acuerdo a la versión de la base de datos GTAP ${ }^{14}$ y el objeto del estudio.

De otra parte, dado el carácter global de la base de datos existe un gran rezago entre el año que se tiene en ésta y el año base utilizado (entre seis u ocho años), dada la obtención y actualización de los datos ${ }^{15}$; por esta razón muchos acuerdos comerciales o cambios arancelarios no pueden verse reflejados en esta base de datos. Para franquear este obstáculo existen dos metodologías, ambas tienen en común una revisión de los acuerdos comerciales suscritos o cambios arancelarios de los países o regiones escogidos para hacer las simulaciones, los cuales se traducen como choques dentro del modelo, sea el GTAPinGAMS o el GTAP, la diferencia radica en la manipulación de los resultados. En el caso de utilizar el modelo GTAP hay una rutina conocida como ALTERTAX, desarrollada por Malcolm (1998), en la cual se introducen estos nuevos aranceles como un choque al modelo, pero la estructura de éste y el valor de los parámetros son elegidos de tal manera que minimicen

14 Por mencionar un caso específico. En América Latina, en la versión 5.0 de GTAP, Bolivia, Ecuador y Perú estaban integrados como una región, posteriormente, en la versión 6.1 se encuentran estos países por separado.

15 Cada dos o tres años el consorcio GTAP actualiza la base de datos a partir de los miembros de la comunidad GTAP. 
la variabilidad de los flujos de valor y, por ello, de la base de datos en su conjunto $^{16}$. En Colombia, al utilizar el modelo GTAPinGAMS, el procedimiento es usar los nuevos aranceles como un choque al modelo, y los resultados obtenidos después de esta simulación son considerados como la nueva base del modelo.

Como se puede apreciar en el Tabla 3, la mayoría de los estudios tienen como año de referencia 1997, tanto para la construcción de la MCS como para la base de datos GTAP (v. 5.0), con excepción de dos modelos: el desarrollado por Bussolo y Roland-Holst (1999), que tiene por objetivo analizar los efectos de la entrada de Colombia en el NAFTA, y el construido por Fedesarrollo (2007), que analiza los efectos del TLC con Estados Unidos sobre la región de Bogotá y Cundinamarca. Hay que tener en cuenta que, a pesar de que la mayoría de los estudios utilizan la misma base de datos, los resultados pueden diferir por tres razones fundamentales: la agregación sectorial, las elasticidades de comercio y producción, y la estructura de producción del modelo.

Adicionalmente, se observa que las elasticidades de comercio, particularmente las Armington, para los modelos de un solo país provienen de la revisión de la literatura, esto es, que se toman a partir de la construcción de otros modelos o de las elasticidades estimadas econométricamente para Colombia ${ }^{17}$. En el caso de los modelos construidos a partir de la base de datos GTAP, las elasticidades son tomadas de Harrison, Rutherford y Tarr (1997), que siguen la "regla de dos"18, esto es, dado que la elasticidad de bienes importados entre diferentes países no está disponible, el valor de esta elasticidad es el doble de la elasticidad entre el bien importado y el bien doméstico para el país de referencia.

16 Véase Schuschny, de Miguel y Duran (2007a; 2007b) para un mayor detalle de esta metodología.

17 Véase sección II.B.

18 Véase Liu, Arndt y Hertel (2004). 
Tabla 3. Datos de los modelos

\begin{tabular}{|c|c|c|c|}
\hline Autores & Año Base & Elasticidades de comercio & Fuente de los datos ${ }^{11}$ \\
\hline $\begin{array}{l}\text { Bussolo y Roland-Holst } \\
\text { (1999) }\end{array}$ & 1992 & $\begin{array}{l}\text { Hernández (1998), Lozano (2004) } \\
\text { y Ramírez, et al. (2004) }\end{array}$ & $\begin{array}{l}\text { MCS para América del } \\
\text { Norte }^{/ 2} \text { y Colombia }\end{array}$ \\
\hline DEE y DDE (2003) & 1997 & GTAP & GTAP v. 5.0 \\
\hline Light y Rutherford (2003) & 1997 & GTAP & GTAP v. 5.0 \\
\hline $\begin{array}{l}\text { Hernández y Sanchez } \\
\text { (2004) }\end{array}$ & 1997 & $\begin{array}{l}\text { Hernández (1998), Lozano (2004) } \\
\text { y Ramírez, et al. (2004) }\end{array}$ & MCS Colombia \\
\hline Light (2004) & 1997 & $\begin{array}{l}\text { Hernández (1998), Lozano (2004) } \\
\text { y Ramírez, et al. (2004) }\end{array}$ & MCS Colombia \\
\hline Botero (2005) & 2000 & $\begin{array}{l}\text { Hernández (1998), Lozano (2004) } \\
\text { y Ramírez, et al. (2004) }\end{array}$ & MCS Colombia \\
\hline López y Botero (2005) & 2000 & $\begin{array}{l}\text { Hernández (1998), Lozano (2004) } \\
\text { y Ramírez, et al. (2004) }\end{array}$ & MCS Colombia \\
\hline Pardo, et al. (2005) & 2001 & GTAP & GTAP v. 6.0 \\
\hline Ramírez y Martín (2005) & 1997 & $\begin{array}{l}\text { Hernández (1998), Lozano (2004) } \\
\text { y Ramírez, et al. (2004) }\end{array}$ & MCS Colombia \\
\hline Vaughan (2005) & 2001 & GTAP & GTAP v. 5.0 \\
\hline Light (2006) & 1997 & GTAP & GTAP v. 5.0 \\
\hline Fedesarrollo (2007) & 2003 & $\begin{array}{l}\text { Hernández (1998), Lozano (2004) } \\
\text { y Ramírez, et al. (2004) }\end{array}$ & $\begin{array}{l}\text { MCS Bogotá y Cundina- } \\
\text { marca }\end{array}$ \\
\hline Gracia y Zuleta (2007) & 2001 & GTAP & GTAP v. 5.0 \\
\hline
\end{tabular}

${ }^{11}$ MCS: Matriz de Contabilidad Social

12 Estados Unidos, Canadá y México

Fuente: elaboración del autor.

\section{Resultados de los modelos}

En cuanto a los efectos encontrados por las diferentes clases de modelos se puede decir que: los resultados de un TLC con Estados Unidos, a nivel macroeconómico (incremento en el crecimiento del PIB) o de bienestar, es marginal, esto se debe a que Colombia contaba con las preferencias del ATPA, que ya de por sí tiene un universo de 5.500 productos -incluyendo confecciones y textiles- con un arancel preferencial, por tanto, los nuevos productos que puedan entrar con un arancel de cero en el mercado norteamericano no llegan a ser más de los que ya se tienen. Sin embargo, la no firma 
Gustavo Hernández: Una revisión de los efectos del Tratado de Libre Comercio...

o la no participación en estos tratados comerciales conlleva consecuencias negativas, tanto a nivel macro como microeconómico, puesto que nuestros competidores, en este caso los países latinoamericanos, obtienen una ventaja para la entrada de sus productos, al no contar Colombia con preferencias arancelarias, ya que las otorgadas por el ATPDEA serían eliminadas.

Los anteriores resultados asumen competencia perfecta por parte de todos los sectores; sin embargo, se puede considerar competencia imperfecta en toda la economía o en algunos sectores en particular. Este tipo de estructura implica que se tengan en cuenta nuevas fuentes de ganancias, tanto en bienestar como en producción, por parte de una liberalización comercial. Por ejemplo, se tiene en cuenta la gran variedad de productos de mayores mercados, con lo cual los consumidores están mucho mejor, y aparecen las economías a escala que ahora son utilizadas, con lo que los precios van a ser menores. Sin embargo, la información debe ser más detallada para el sector que tenga competencia imperfecta, solo por citar las más frecuentes: estimaciones de markup o de elasticidades de demanda, economías de escala, intensidad de la competencia.

Dentro de los modelos de competencia imperfecta se encuentran los construidos por Light (2004; 2006) y Ramírez y Martín (2005), que han considerado la reducción de las barreras no arancelarias, exclusivamente en el sector de servicios. La eliminación de este tipo de barreras trae como consecuencia un importante impacto macroeconómico, gracias al impulso que se le da a la inversión extranjera directa, ya que el crecimiento es de 3,6\% del PIB en el mediano plazo y hasta de 5,8\% en el largo plazo (Light, 2004). Estos resultados son mucho más modestos para Ramírez y Martín (2005), ya que son de una magnitud de $1,1 \%$. En términos de bienestar hay un incremento equivalente a la mitad del crecimiento del PIB, como consecuencia de una mayor entrada de inversión extrajera directa a la economía y al incremento de la competencia de las firmas domésticas con las extrajeras. Esto puede llevar a la salida de empresas domésticas, pero el empleo no se vería tan afectado, ya que esta fuerza laboral es absorbida por la creación y entrada de nuevas empresas.

En todos los modelos, dependiendo de la desagregación sectorial de cada uno de estos, se encuentra que los sectores más beneficiados con esta política 
son los que se encuentran agrupados en el sector industrial, mientras que sobre el sector agrícola se presenta un impacto negativo. Por estas razones, las acciones políticas se han encaminado a que el sector agrícola sea más competitivo, a través de subsidios o por medio de un trato preferencial dentro de la negociación.

Otro de los aspectos estudiados dentro de la evaluación del TLC con Estados Unidos, han sido los impactos sobre la pobreza y la distribución del ingreso. Este mecanismo depende mucho de la distribución del ingreso en la población, las fuentes de ingreso en sus diferentes niveles, la reacción de los agentes ante los choques de política, entre otros. Hernández y Sanchez (2004) y Pardo, et al. (2005), encuentran que las políticas comerciales tienen como consecuencia una reducción de la pobreza y un leve mejoramiento en la distribución del ingreso, debido a que el aumento de los precios de la economía (efecto sustitución) se ve más que compensado por el incremento en los salarios (efecto ingreso), vía un aumento del crecimiento económico.

Aunque no se ha considerado la dinámica en estos modelos, se puede decir que estos son de largo plazo, ya que consideran que el trabajo es perfectamente móvil entre sectores ${ }^{19}$. Para considerar las implicaciones sobre los salarios, es importante tener en cuenta que al cambiar los precios relativos se incrementan en los sectores productivos, en los que la ventaja comparativa es mayor, una especialización. Por tanto, una mejor reasignación de recursos hace que los salarios en promedio se incrementen, lo cual llevaría a un incremento de los ingresos de los hogares. En consecuencia, dependiendo de cómo sea la estructura del mercado laboral, esto es, qué tan flexible o inflexible se considere (competencia perfecta, salario mínimo, salarios de eficiencia, migración), los resultados sobre los ingresos y, por tanto, la distribución, pueden arrojar diferentes resultados.

Aunque en una menor medida, se ha tratado de hacer un esfuerzo en evaluar los impactos de la firma del TLC con Estados Unidos a nivel regional, como el modelo construido por Fedesarrollo (2007) para la región de Bogotá

19 Para simular el corto plazo se puede hacer el supuesto de que los factores de producción son inmóviles entre sectores. 
y Cundinamarca. Ellos encuentran que, ante una firma del TLC con Estados Unidos, los ganadores con esta política arancelaria son los sectores industriales, ya que tienen un mayor comercio con Estados Unidos; y el sector de servicios financieros, por la demanda potencial que tiene este sector por parte de las demás regiones del país.

\section{Comentarios finales}

Los MEGC proveen una estructura rigurosa y teóricamente consistente para el análisis de políticas comerciales. Esto no quiere decir que no se cuente con otro tipo de herramientas, como lo son los modelos de gravedad o de equilibrio parcial. Los resultados numéricos pueden ser interpretados más como un indicador de hacia dónde van los impactos, que las magnitudes reales de las políticas. Como se puede ver en los ejercicios de simulación de la firma de un TLC con Estados Unidos, las magnitudes cambian ligeramente, en algunos $\operatorname{casos}^{20}$, pero los resultados cualitativos se mantienen.

Los resultados de las distintas simulaciones en estos modelos están marcados por: i) las bases de datos utilizadas, ii) los parámetros (particularmente elasticidades de comercio) del modelo y iii) las características teóricas del modelo. La base de datos con que se han construido los diferentes modelos, conocida como MCS, afecta los resultados, en el sentido de que dependiendo de la agregación se pueden identificar mejor los "ganadores" o "perdedores" ante la firma de un TLC. Por ello, las elasticidades Armington juegan un papel esencial en la determinación de las ganancias de bienestar del modelo, ante menores elasticidades las ganancias esperadas son menores, por esto la elección de ellas debe realizarse con máximo cuidado. Finalmente, las características del modelo dan como resultado grandes cambios en los resultados del modelo, al considerar alternativas diferentes de ver el mundo (competencia perfecta vs. competencia imperfecta).

La validación expost-esto es contrastar los resultados después de la firma de la entrada efectiva del TLC con Estados Unidos con los resultados

20 Modelos con características similares. 
alcanzados- de estos modelos puede mejorar la especificación del modelo y ayudar a observar cuáles son las especificaciones de modelación más adecuadas para emplear en los diferentes escenarios de simulación.

\section{Bibliografía}

Anderson, Kym; van der Mensbrugghe, Dominique \& Martin, Will (2005). "Market and welfare implications of Doha reform scenarios". En: Trade reform and the Doha Agenda (233-399). Washington D.C: The World Bank.

Anderson, Kym; van der Mensbrugghe, Dominique \& Martin, Will (2006). "Doha merchandise trade reform: What's at stake for developing Countries?”, Policy Research Working Paper, No. 3848. The World Bank

Armington, Paul (1969). "A theory of demand for products distinguished by place of production”, IMF Staff Papers, Vol. 16, Issue 1, pp. 159 - 176.

Botero, Jesús (2005). "Estimación del impacto sobre el empleo de los tratados de libre comercio en Colombia: Análisis de equilibrio general computable", Estudios y Perspectivas, No 8. CEPAL.

Boü̈T, Antoine (2008). "The expected benefits of trade liberalization for world income and development: Opening the "black box" of global trade modeling”, Food Policy Review, No 8. International Food Policy Research Institute.

Bourguignon, François; Robilliard, Anne-Sophie \& Robinson, Sherman (2005). "Representative versus real households in the macroeconomic modeling of inequality". En: Kehoe, Timothy; Srinivasan, T \& Whalley, Jhon (Eds.), Frontiers in applied general equilibrium modeling (219-254). New York: Cambridge University Press.

Bussolo, Mauricio \& Roland-Holst, David (1999). "Colombia y el TLCAN", Integración y Comercio, Vol. 3, No. 9, pp. 27 - 61.

Cárdenas, Mauricio \& García, Camilo (2004). "El modelo gravitacional de comercio y el TLC entre Colombia y Estados Unidos", Documento de Trabajo, No 27. Fedesarrollo 
Gustavo Hernández: Una revisión de los efectos del Tratado de Libre Comercio...

Devarajan, Shantayanan; Go, Delfin \& Li, Hongyi (1999). "Quantifying fiscal effects of trade reform”. Working Paper, No 2162. The World Bank,

Dirección de Estudios Económicos y Dirección de Desarrollo EmPRESARIAL (DEE - DDE) (2003). "Efectos de un acuerdo bilateral de libre comercio con Estados Unidos”. Archivos de Economía, No 229. Departamento Nacional de Planeación.

Fedesarrollo (2007). Impacto económico del TLC con Estados Unidos en la región Bogotá-Cundinamarca. Bogotá: Cámara de Comercio de Bogotá.

Gracia, Orlando \& Zuleta, Hernando (2007). “Tratado de libre comercio entre Colombia y Estados Unidos: ¿Qué impacto puede tener en Colombia?”. Documento de Trabajo, No 29. Universidad del Rosario

Harrison, Glenn; Rutherford, Thomas \& Tarr, David (1997). "Quantifying the Uruguay Round”, The Economic Journal, Vol. 107, No. 444, pp. $1405-1430$.

Harrison, Glenn; Rutherford, Thomas \& Tarr, David (2003). "Trade liberalization, poverty and efficient equity", Journal of Development Economics, Vol. 71, Issue 1, pp. 97 - 128.

Hernández, Gustavo (1998). "Elasticidades de sustitución de las importaciones para la economía colombiana", Revista de Economía de la Universidad del Rosario, Vol. 1, No. 2, pp. 79 - 89.

Hernández, Gustavo \& Sánchez, Fabio (2004). "Colombia: aumento de las exportaciones y sus efectos sobre el crecimiento, empleo y pobreza”, Desarrollo y Sociedad, No. 53, pp. 193 - 226.

Hertel, Thomas (Ed.) (1999). Global trade analysis: Modeling and applications. Purdue, Indiana: Cambridge University Press.

Hertel, Thomas; Hummels, David; Ivanic, Maros \& Keeney, Roman (2004). "How confident can we be in CGE-based assessments of free trade agreements?". NBER Working Paper, No 10477. National Bureau of Economic Research. 
Light, Miles (2004). "FTAA and Service Liberalization in Colombia”, Desarrollo y Sociedad, No. 53, pp. 165 - 192.

Light, Miles (2006). “Liberalización del sector servicios”. En: Comunidad Andina, Secretaría General (Eds.) Desarrollo, Negociaciones hemisféricas y la comunidad andina: Evaluación de impacto (pp. 129 - 168). Lima: Comunidad Andina, Banco Interamericano de Desarrollo.

Light, Miles \& Rutherford, Thomas (2003). "Free trade area of the Americas: An impact assessment for Colombia", Archivos de economía, No 222. Departamento Nacional de Planeación.

Lipsey, R. \& Lancaster, Kelvin (1956). "The General Theory of Second Best”, Review of Economic Studies, Vol. 24, No. 1, pp. 11-32.

Liu, Jing; Arndt, Channing \& Hertel, Thomas. (2004). "Parameter Estimation and Measures of Fit in a Global, General Equilibrium Model", Journal of Economic Integration, Vol. 19, No. 3, pp. 626-649.

Lofgren, Hans; Lee, Rebecca \& Robinson, Sherman (2002). "A standard computable general equilibrium (CGE) model in GAMS”, Microcomputers in policy research, No 5. International Food Policy Research Institute.

López, Hugo \& Botero, Jesús (2005). "Impactos del TLC sobre el empleo", Planeación y Desarrollo, Vol. XXXVI, No. 1, pp. 77 - 97.

Lozano, Carolina (2004). "Elasticidades de sustitución Armington para Colombia”, Archivos de Economía, No 271. Departamento Nacional de Planeación.

Malcolm, Gerard (1998). "Adjusting Tax Rates in the GTAP Data Base”, Technical Paper, No 12. University of Purdue.

McDaniel, Christine \& Balistreri, Edward (2003). "Una revisión de las elasticidades de sustitución Armington", Integración y comercio, No 18, pp. 171-184.

Pardo, Oliver; Lozano, Carolina; Perdomo, Álvaro \& Delgado, Catalina (2005). "Colombia y el TLC: Efectos sobre la distribución del ingreso y 
Gustavo Hernández: Una revisión de los efectos del Tratado de Libre Comercio...

la pobreza", Archivos de Economía, Documento No 289. Departamento Nacional de Planeación.

Perdomo, Álvaro (2008). "Modelo estándar de equilibrio general computable”, Archivos de Economía, Documento No 342. Departamento Nacional de Planeación.

Ramírez, Juan \& Martín, Clara (2005). "El impacto económico de un acuerdo parcial de libre comercio entre Colombia y Estados Unidos", Planeación y Desarrollo, Vol. XXXVI, No. 1, pp. 23 - 76.

Ramírez, Manuel; Martínez, Héctor; Ortiz, Ximena; González, Fredy \& Barrios, Camilo (2004). "Respuestas de la oferta y la demanda agrícola en el marco de un TLC con Estados Unidos", Documento de Trabajo, No 49. Observatorio Agrocadenas Colombia.

Rutherford, Thomas \& PAltsev, Sergey (2000). "GTAPinGAMS and GTAP-EG: Global datasets for economic research and illustrative models". Working Paper. Deparment of Economics, University of Colorado.

Schuschny Andrés; De Miguel, Carlos \& Durán, José (2007a). “Acuerdos de libre comercio entre los países andinos y los Estados Unidos: ¿Cuánto se puede esperar de ellos?”, Serie Comercio Internacional, No 77. CEPAL.

Schuschny Andrés; De Miguel, Carlos \& Durán, José (2007b). “El modelo GTAP y las preferencias arancelarias en América Latina y el Caribe: reconciliando su año base con la evolución reciente de la agenda de liberalización regional”. Serie Manuales, No 53. CEPAL.

Umaña, Camilo (2011). "Una evaluación de la estrategia comercial de Colombia a la luz de un Modelo de Equilibrio General Computable basado en la ecuación de gravedad". Archivos de Economía, Documento No 379. Departamento Nacional de Planeación.

VAughan, Daniel (2005). “Tratado de libre comercio y barreras no arancelarias: Un análisis crítico”, Planeación y Desarrollo, Vol. XXXVI, No. 1, pp. 99 - 162. 


\section{Apéndice.}

\section{Resultados de las estimaciones de las elasticidades Armington}

Tabla 4. Elasticidades Armington, series de tiempo

\begin{tabular}{lccc}
\hline \multicolumn{1}{c}{ Método de Estimación } & Elasticidad & t-student & $\begin{array}{c}\text { Número de } \\
\text { observaciones }\end{array}$ \\
\hline OLS sin tendencia & 1.20 & 5.81 & 27 \\
OLS con tendencia & 1.13 & 5.35 & 27 \\
OLS con PIB & 1.14 & 5.39 & 27 \\
SUR con PIB, disponible todos los años & 1.30 & 6.97 & 27 \\
SUR con tendencia, disponible todos los años & 1.31 & 6.98 & 27 \\
\hline
\end{tabular}

Fuente: Devarajan, Go y Li (1999).

Tabla 5. Elasticidades Armington, series de tiempo - cointegración

\begin{tabular}{lcc}
\hline & \multicolumn{2}{c}{ Elasticidades } \\
\hline Corto plazo & Largo plazo \\
\hline Pecuario & 0.267 & 0.299 \\
& $(0.101)$ & $(0.117)$ \\
Minería & 0.894 & 1.448 \\
& $(0.255)$ & $(0.196)$ \\
Carne y pescado & 0.622 & 0.748 \\
& $(0.165)$ & $(0.283)$ \\
Alimentos procesados & 0.287 & 0.321 \\
Telas y tejidos & $(0.120)$ & $(0.550)$ \\
Confecciones & 0.344 & 0.466 \\
Caucho y plástico & $(0.152)$ & $(0.265)$ \\
& 0.220 & 0.378 \\
Refinados de petróleo & $(0.108)$ & $(0.219)$ \\
& 0.495 & -0.135 \\
& $(0.222)$ & $(0.493)$ \\
& 0.541 & 0.960 \\
& $(0.137)$ & $(0.318)$ \\
& 0.460 & 1.134 \\
& $(0.202)$ & $(1.184)$
\end{tabular}

(Continúa) 
Gustavo Hernández: Una revisión de los efectos del Tratado de Libre Comercio...

Tabla 5. (Continuación)

\begin{tabular}{lcc}
\hline & \multicolumn{2}{c}{ Elasticidades } \\
\hline Corto plazo & Largo plazo \\
\hline \multirow{2}{*}{ Productos metálicos } & 0.420 & 0.219 \\
& $(0.496)$ & $(0.209)$ \\
Maquinaria y equipo & 0.496 & -0.972 \\
& $(0.195)$ & $(1.157)$ \\
Equipo de transporte & 0.810 & 2.135 \\
& $(0.202)$ & $(0.693)$ \\
Otras industrias & 0.263 & 0.500 \\
& $(0.110)$ & $(0.268)$ \\
& 0.717 & 1.670 \\
\end{tabular}

Nota: Error estándar entre paréntesis.

Fuente: Lozano (2004).

Tabla 6. Elasticidades Armington, series de tiempo (1970 - 1994)

\begin{tabular}{lcc}
\hline & $\begin{array}{c}\text { Mínimos cuadrados } \\
\text { ordinarios }\end{array}$ & $\begin{array}{c}\text { Modelo de } \\
\text { ajuste parcial }\end{array}$ \\
\hline Agricultura sin café & 0.624 & 0.538 \\
Minería y petróleo & $(3.109)$ & $(2.996)$ \\
Alimentos manufacturados & -1.487 & -0.715 \\
Bienes de consumo liviano & $(-2.573)$ & $(-2.615)$ \\
& 0.131 & 0.157 \\
Bienes de consumo intermedio & $(0.543)$ & $(0.860)$ \\
Bienes de capital & 0.857 & 0.169 \\
Comercio & $(4.006)$ & $(1.242)$ \\
Transporte & 0.304 & 0.137 \\
& $(3.028)$ & $(1.390)$ \\
& 0.237 & 0.213 \\
& $(1.038)$ & $(1.707)$ \\
& 0.380 & 0.563 \\
& $(0.874)$ & $(1.287)$ \\
& 0.233 & 0.214 \\
& $(0.982)$ & $(3.992)$ \\
\hline
\end{tabular}

Nota: t-student entre paréntesis.

Fuente: Hernández (1998). 
Tabla 7. Elasticidades Armington para el sector agropecuario

\begin{tabular}{lcc}
\hline \multicolumn{1}{c}{ Producto } & Período & Elasticidad \\
\hline Aceite de origen vegetal refinado & $1975-2002$ & -1.07 \\
& $1998-2002$ & -1.05 \\
Otros aceites y grasas & $1975-2002$ & -1.53 \\
Frijol & $1998-2002$ & -1.37 \\
& $1975-2002$ & -0.37 \\
Harina de trigo & $1998-2002$ & -0.55 \\
& $1975-2002$ & -1.5 \\
Maíz & $1998-2002$ & -1.42 \\
Huevos con cascara, frescos y conservados & $1975-2002$ & -0.95 \\
& $1998-2002$ & -0.95 \\
Filetes de pescado y otros & $1975-2002$ & -1.73 \\
Ganado bovino & $1998-2002$ & -1.69 \\
Algodón desmotado & $1975-2002$ & -1.64 \\
Trigo en grano & $1998-2002$ & -1.27 \\
Ganado porcino & $1975-2002$ & -1.49 \\
Arroz, blanqueado, pulido, semielaborado o elaborado & $1998-2002$ & -2.35 \\
Cacao incluso tostado & $1975-2002$ & -2.17 \\
& $1998-2002$ & -1.65 \\
& $1998-2002$ & -1.89 \\
& $1975-2002$ & -1.38 \\
& $1975-2002$ & -1.99 \\
& $1998-2002$ & -0.95 \\
& $1975-2002$ & -1.58 \\
& $1975-2002$ & -1.06 \\
& $1998-2002$ & -1.04 \\
\hline
\end{tabular}

Nota: elasticidades determinadas como en un sistema AIDS.

Fuente: Ramírez, et al. (2004). 